\title{
Corrigendum to "Combining Galerkin approximation techniques with the principle of Hashin and Shtrikman to derive a new FFT-based numerical method for the homogenization of composites" [Comput. Methods Appl. Mech. Engrg. 217-220 (2012) 197-212]
}

\author{
S. Brisard ${ }^{\mathrm{a}, *}$, L. Dormieux ${ }^{\mathrm{a}}$
}

${ }^{a}$ Université Paris-Est, Laboratoire Navier (UMR 8205), CNRS, ENPC, IFSTTAR, F-77455 Marne-la-Vallée

Assumption 1 in our original paper can be replaced with the following, less stringent assumption.

Assumption 1. There exists $\lambda>0$ such that at any point $\mathbf{x} \in \Omega$, the eigenvalues of $\left[\mathbf{C}(\mathbf{x})-\mathbf{C}_{0}\right]$ are greater than $\lambda$ in absolute value.

Proof of Theorem 4 requires that the local stiffness be bounded from below and above. Therefore, Assumption 2 must be altered as follows

Assumption 2. There exists $\kappa_{\max }>\kappa_{\min }>0$ and $\mu_{\max }>\mu_{\min }>$ 0 such that at any point $\mathbf{x} \in \Omega$

$\kappa_{\min } \leq \kappa(\mathbf{x}) \leq \kappa_{\max }, \quad \mu_{\min } \leq \mu(\mathbf{x}) \leq \mu_{\max }$.

Then, the end of the proof of Theorem 4 (starting from "Taking advantage of the isotropy") must be modified as follows.

Proof of Theorem 4. [...] Taking advantage of the isotropy of both local and reference materials, the above volume averages can be expanded

$$
\begin{aligned}
|\Omega| \overline{\tau^{+}:\left(\mathbf{C}-\mathbf{C}_{0}\right)^{-1}: \boldsymbol{\tau}^{+}}= & \int_{\kappa(\mathbf{x})>\kappa_{0}} \frac{\left\|\boldsymbol{\tau}^{\mathrm{hyd}}(\mathbf{x})\right\|^{2}}{d\left[\kappa(\mathbf{x})-\kappa_{0}\right]} \mathrm{d} \Omega \\
& +\int_{\mu(\mathbf{x})>\mu_{0}} \frac{\left\|\tau^{\mathrm{dev}}(\mathbf{x})\right\|^{2}}{2\left[\mu(\mathbf{x})-\mu_{0}\right]} \mathrm{d} \Omega,
\end{aligned}
$$

and

$$
\begin{aligned}
|\Omega| \overline{\tau^{-}: \mathbf{S}_{0}:\left(\mathbf{S}-\mathbf{S}_{0}\right)^{-1}: \mathbf{S}_{0}: \boldsymbol{\tau}^{-}}= & \int_{\kappa(\mathbf{x})<\kappa_{0}} \frac{\kappa(\mathbf{x})\left\|\boldsymbol{\tau}^{\mathrm{hyd}}(\mathbf{x})\right\|^{2}}{d \kappa_{0}\left[\kappa_{0}-\kappa(\mathbf{x})\right]} \mathrm{d} \Omega \\
& +\int_{\mu(\mathbf{x})<\mu_{0}} \frac{\mu(\mathbf{x})\left\|\tau^{\mathrm{dev}}(\mathbf{x})\right\|^{2}}{2 \mu_{0}\left[\mu_{0}-\mu(\mathbf{x})\right]} \mathrm{d} \Omega .
\end{aligned}
$$

From Assumption 2, we first have

$$
\begin{aligned}
&|\Omega| \overline{\boldsymbol{\tau}^{+}:\left(\mathbf{C}-\mathbf{C}_{0}\right)^{-1}: \boldsymbol{\tau}^{+}} \geq \frac{\int_{\kappa(\mathbf{x})>\kappa_{0}}\left\|\boldsymbol{\tau}^{\mathrm{hyd}}(\mathbf{x})\right\|^{2} \mathrm{~d} \Omega}{d\left(\kappa_{\max }-\kappa_{0}\right)} \\
&+\frac{\int_{\mu(\mathbf{x})>\mu_{0}}\left\|\boldsymbol{\tau}^{\mathrm{dev}}(\mathbf{x})\right\|^{2} \mathrm{~d} \Omega}{2\left(\mu_{\max }-\mu_{0}\right)},
\end{aligned}
$$

\footnotetext{
${ }^{*}$ Corresponding author.

Email addresses: sebastien.brisard@ifsttar.fr (S. Brisard), luc.dormieux@enpc.fr (L. Dormieux)
}

then

$$
\begin{aligned}
& |\Omega| \overline{\boldsymbol{\tau}^{-}: \mathbf{S}_{0}:\left(\mathbf{S}-\mathbf{S}_{0}\right)^{-1}: \mathbf{S}_{0}: \boldsymbol{\tau}^{-}} \geq \frac{\kappa_{\min } \int_{\kappa(\mathbf{x})<\kappa_{0}}\left\|\boldsymbol{\tau}^{\mathrm{hyd}}(\mathbf{x})\right\|^{2} \mathrm{~d} \Omega}{d \kappa_{0}\left(\kappa_{0}-\kappa_{\min }\right)} \\
& +\frac{\mu_{\min } \int_{\mu(\mathbf{x})<\mu_{0}}\left\|\tau^{\mathrm{dev}}(\mathbf{x})\right\|^{2} \mathrm{~d} \Omega}{2 \mu_{0}\left(\mu_{0}-\mu_{\min }\right)} .
\end{aligned}
$$

from which the following bound results

$a(\boldsymbol{\tau}, \boldsymbol{\varpi}) \geq \frac{\alpha}{|\Omega|} \int_{\Omega}\left[\left\|\tau^{\mathrm{hyd}}(\mathbf{x})\right\|^{2}+\left\|\tau^{\mathrm{dev}}(\mathbf{x})\right\|^{2}\right] \mathrm{d} \Omega=\alpha\|\boldsymbol{\tau}\|_{\mathbb{V}}^{2}$,

with $^{1}$

$$
\begin{aligned}
& \alpha=\min \{ {\left[d\left(\kappa_{\max }-\kappa_{0}\right)\right]^{-1},\left[2\left(\mu_{\max }-\mu_{0}\right)\right]^{-1} } \\
&\left.\kappa_{\min }\left[d \kappa_{0}\left(\kappa_{0}-\kappa_{\min }\right)\right]^{-1}, \mu_{\min }\left[2 \mu_{0}\left(\mu_{0}-\mu_{\min }\right)\right]^{-1}\right\} .
\end{aligned}
$$

The proof of the first statement is complete, since $\|\varpi\|_{\mathbb{V}}=\|\tau\|_{\mathbb{V}}$, and $\alpha>0$.

\footnotetext{
${ }^{1}$ In this definition of $\alpha$, it is assumed that $\kappa_{\min }<\kappa_{0}<\kappa_{\max }$ and $\mu_{\min }<\mu_{0}<$ $\mu_{\max }$. The proof remains valid if any of these inequalities are not verified. For example, if $\mu_{\min } \geq \mu_{0}$, then, from Assumption $2, \mu(\mathbf{x}) \geq \mu_{0}$ at any point $\mathbf{x} \in \Omega$ In other words, the integral over the set of points $\mathbf{x} \in \Omega$ such that $\mu(\mathbf{x})<\mu_{0}$ is null. Then Eq. (49) still holds, provided that $\alpha$ is defined as follows

$\alpha=\min \left\{\left[d\left(\kappa_{\max }-\kappa_{0}\right)\right]^{-1},\left[2\left(\mu_{\max }-\mu_{0}\right)\right]^{-1}, \kappa_{\min }\left[d \kappa_{0}\left(\kappa_{0}-\kappa_{\min }\right)\right]^{-1}\right\}$.
} 